\title{
Conventional Synthesis, In Vitro Antimicrobial Activity and Calculation of Pharmacokinetic Properties of Thioether Derivatives of Quinoxaline
}

\author{
RICHA SAHU* and S. P. SHRIVASTAVA \\ Department of Chemistry, Synthetic Organic Chemistry Laboratory, Dr. Hari Singh \\ Gour(Central) University, Sagar(M.P)- 470003, India \\ richasahu776@gmail.com
}

Received 13 December 2015 / Accepted 11 January 2016

\begin{abstract}
Different thioether derivatives of quinoxaline moiety have been synthesized by conventional method. All the prepared compounds were analyzed by IR and NMR spectral analysis followed by antimicrobial screening using disk diffusion (Kirby Baeur) method against pathogenic bacteria and fungi. Ciprofloxacin and fluconazole were used as a standard drug for the study. Among them compound $\mathbf{3 i}$ shows better activity in comparison with standard.
\end{abstract}

Keywords: Quinoxaline, Antimicrobial activity, Pharmacokinetic property

\section{Introduction}

Heterocyclic compounds symbolize imperative set of biologically active dynamic molecules ${ }^{1}$. Among the diverse classes of nitrogen having heterocyclic compounds, quinoxaline derivatives are the essential components of numerous pharmacologically active compounds $^{2-7}$. Quinoxaline exhibit a wide variety of biological activity ${ }^{8}$ which has made them advantage structures in combinatorial drug discovery libraries ${ }^{9}$ quinoxaline and its derivatives are very important part of numerous bioactive molecules which locate function as antimicrobial ${ }^{10}$, anticancerous ${ }^{11}$, antidepressant ${ }^{12,13}$ antitubercular $^{14}$ anticonvulsant $^{15}$, analgesic ${ }^{16}$, anti-inflammatory ${ }^{17}$ anti-platelet ${ }^{18}$, anti-viral ${ }^{19}$, anti-tumoral ${ }^{20}$ and anti-malaria ${ }^{21}$ activities.

Quinoxaline ring is a part of diverse antibiotics, for instance Hinomycin, Levomycin and actinoleutin $^{22,23}$. The quinoxaline is exemplified as a bioesters of quinoline, naphthalene, benzothiophene and other aromatic ring for example pyridine and pyrazine. It is one of the most significant modules of known antagonists of amino propanoic acid (AMPA). The quinoxaline moiety is too present in peptide antibiotics. Quinoxaline derivatives are double nitrogen containing heterocyclic compounds which have biological consequence. 


\section{Experimental}

The entire chemicals and reagents in latest study were of AR grade and acquired from Merk(India). The reactions were observed by thin layer chromatography(TLC) on Merk precoated silica GF254 plates and envisaged under UV-chamber.Melting points (uncorrected) were concluded by open capillary tubes method. IR spectra $\left(\lambda_{\max }\right.$ in $\left.\mathrm{cm}^{-1}\right)$ were accomplished on a Shimadzu FTIR 8300S spectrophotometer with $\mathrm{KBr}$ pellets. ${ }^{1} \mathrm{H}$ NMR spectra were verified on a Bruker Avance II 400NMR spectrometer instrument with DMSO as solvent (chemical shift in ppm) by means of TMS as the internal standard. The elemental analysis $(\mathrm{C}, \mathrm{H}, \mathrm{N})$ of the synthesized compounds were performed on thermo scientific (FLASH 2000) CHN Elemental Analyser. This is microprocessor based instrument which determines the $\mathrm{CHN}$ Analyzer percentages of $\mathrm{C}, \mathrm{H}$ and $\mathrm{N}$ with an accuracy of $0.3 \%$.

\section{Synthesis of Compound $\mathbf{3 a - 3 h}$}

The different thioether derivatives of (substituted phenyl)-2-(tetrazolo [1, 5-a] quinoxaline-4ylthio) acetamide (3a-3h) were synthesized (Scheme 1), characterized by spectral analysis as per literature method ${ }^{24}$.

\section{Results and Discussion}

TPSA topological polar surface area is a tool for prediction of drug transport properties. TPSA was used to calculate the percentage of absorption (\% ABS) by the following equation $\% \mathrm{ABS}=109 \pm 0.345 \times \mathrm{TPSA}$. A computional study for prediction of ADME properties of all the compounds presented in Table 1.<smiles>[R]Oc1ccccc1NSc1nc2ccccc2n2nnnc12</smiles>

tetrazolo[1,5a]quinoxaline

sodium tetrazolo[1,5- $a$ ]quinoxaline-4-thiolate<smiles>[R]c1ccc(NC(=O)CSc2nc3ccccc3n3nnnc23)cc1</smiles>

(Substituted phenyl)-2-(tetrazolo[1,5-a]quinoxline-4ylthio)acetamide

\section{(3a-3I)}

Scheme 1. Synthetic route for the preparation of compounds 
Table 1. Pharmacokinetic parameters important for good oral bioavailability of title compounds (3a-31)

\begin{tabular}{|c|c|c|c|c|c|c|c|c|c|c|}
\hline 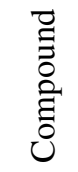 & $\simeq$ & $\begin{array}{l}\mathscr{2} \\
\frac{0}{2} \\
0\end{array}$ & 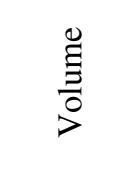 & $\begin{array}{l}\overleftarrow{\curvearrowleft} \\
\text { مै }\end{array}$ & $\begin{array}{l}n \\
0 \\
\\
z\end{array}$ & 㟔 & $\stackrel{\theta}{\underline{I}}$ & $\begin{array}{l}0 \\
0 \\
0 \\
1\end{array}$ & $\sum$ & 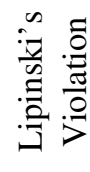 \\
\hline Rule & & - & - & - & - & $<10$ & $<5$ & $\leq 5$ & $<500$ & $\leq 1$ \\
\hline $3 \mathbf{a}$ & $2-\mathrm{NO}_{2}$ & 63.838 & 301.827 & 130.905 & 5 & 10 & 1 & 2.662 & 381.377 & 0 \\
\hline $3 \mathbf{b}$ & $3-\mathrm{NO}_{2}$ & 63.838 & 301.827 & 130.905 & 5 & 10 & 1 & 2.686 & 381.377 & 0 \\
\hline $3 c$ & $3-\mathrm{Cl}$ & 79.648 & 292.029 & 85.081 & 4 & 7 & 1 & 3.405 & 370.825 & 0 \\
\hline $3 d$ & 4-Cl & 79.648 & 292.029 & 85.081 & 4 & 7 & 1 & 3.429 & 370.825 & 0 \\
\hline $3 e$ & $2-\mathrm{CH}_{3}$ & 79.648 & 295.054 & 85.081 & 4 & 7 & 1 & 3.152 & 350.407 & 0 \\
\hline $3 f$ & $4-\mathrm{CH}_{3}$ & 79.648 & 295.054 & 85.081 & 4 & 7 & 1 & 3.2 & 350.407 & 0 \\
\hline $3 g$ & $2-\mathrm{OCH}_{3}$ & 76.462 & 304.039 & 94.315 & 5 & 8 & 1 & 2.76 & 366.406 & 0 \\
\hline $3 h$ & $4-\mathrm{OCH}_{3}$ & 76.462 & 366.406 & 94.315 & 5 & 8 & 1 & 2.808 & 366.406 & 0 \\
\hline $3 \mathbf{i}$ & $2-\mathrm{Cl}$ & 79.647 & 292.03 & 85.08 & 4 & 7 & 1 & 3.38 & 370.82 & 0 \\
\hline $3 \mathbf{j}$ & $3-\mathrm{Cl}, 4-\mathrm{F}$ & 79.647 & 296.96 & 85.08 & 4 & 7 & 1 & 3.52 & 388.81 & 0 \\
\hline $3 k$ & $2,3-\mathrm{CH}_{3}$ & 79.648 & 305.56 & 85.08 & 4 & 7 & 1 & 4.01 & 405.27 & 0 \\
\hline 31 & 4-Br & 79.648 & 296.379 & 85.081 & 4 & 7 & 1 & 3.56 & 415.276 & 0 \\
\hline
\end{tabular}

\%ABS:Percentage of absorption; TPSA: Topological polar surface area; NROTB: Number of rotatable bonds; MW: Molecular weight; LogP: Logarithm of compound partition coefficient between n-octanol and water; HBA: Number of hydrogen bond donors; HBD: Number of hydrogen bond acceptors

Synthesis of N-(Substituted phenyl)-2-(tetrazolo [1,5-]quinoxaline-4-ylthio) acetamide-3(i-l)

Equimolar amount of sodium tetrazolo[1,5-a]quinoxaline-4-thiolate(0.01) and various 2-chloro- $N$-(Substituted phenyl)-acetamides (0.01) were added in DMF and heated under reflux for 8-10 h. The purity of the compounds was checked by TLC with toluene: acetone 8:2 as mobile phase. The mixture was kept at room temperature and relocated into ice chilled water. The solid was separated out by filteration, rinsed with water and dried out, further purified through recrystallization from ethanol.

$\mathrm{N}$-(2-Chlorophenyl)-2-(tetrazolo[1,5-]quinoxaline-4-ylthio)acetamide (3i)

Elemental Analysis \% found C 50.39,H 2.91,N 25.71 Calc C 50.30,H 2.85,N 25.65 FT$\mathrm{IR}(\mathrm{KBr})$, in $\mathrm{cm}^{-1} 3220(\mathrm{~N}-\mathrm{H}), 3040(\mathrm{C}-\mathrm{H}), 2880\left(-\mathrm{CH}_{2^{-}}, 1660(\mathrm{C}=\mathrm{O}), 1630(\mathrm{C}=\mathrm{N}), 1420\right.$ and 1250(-C-S-CH $\left.{ }_{2}\right), 1050(\mathrm{C}-\mathrm{Cl})$ aryl chloride and 680(- $\left.\mathrm{CH}_{2}-\mathrm{S}\right) .{ }^{1} \mathrm{H}$ NMR (DMSO-d6,400 MHZ, ppm) $2.39\left(\mathrm{~s}, 3 \mathrm{H}, \mathrm{CH}_{3}\right), 4.165\left(\mathrm{~s}, 2 \mathrm{H}, \mathrm{CH}_{2}\right), 7.118-8.034(\mathrm{~m}, 8 \mathrm{H}, \mathrm{Ar}-\mathrm{H})$ and $8.495(\mathrm{~s}, 1 \mathrm{H}, \mathrm{N}-\mathrm{H})$.

$\mathrm{N}$-(3-Chloro-4-fluorophenyl)-2-(tetrazolo[1,5-a]quinoxaline-4-ylthio) acetamide (3j)

Elemental analysis\%found C 50.39,H 2.91,N 25.71 Cal C 50.32,H 2.81,N 25.61,FT-IR(KBr) in $\mathrm{cm}^{-1} 3250(\mathrm{~N}-\mathrm{H}), 3040(\mathrm{C}-\mathrm{H}), 2890\left(-\mathrm{CH}_{2}-\right), 1650(\mathrm{C}=\mathrm{O}), 1595(\mathrm{C}=\mathrm{N}), 1340$ and $1250(-\mathrm{C}-\mathrm{S}-$ $\mathrm{CH} 2$,), $1040(\mathrm{C}-\mathrm{Cl})$ aryl chloride and $660\left(\mathrm{CH}_{2}-\mathrm{S}\right) .{ }^{1} \mathrm{H}$ NMR(DMSO-d6, $400 \mathrm{MHZ}$ ppm) 2.637(s,3H, $\left.\mathrm{CH}_{3}\right), 4370\left(\mathrm{~s}, 2 \mathrm{H}, \mathrm{CH}_{2}\right), 7.068-8.022(\mathrm{~m}, 8 \mathrm{H}, \mathrm{Ar}-\mathrm{H})$ and $9.648(\mathrm{~s}, 1 \mathrm{H}, \mathrm{N}-\mathrm{H})$.

$N$-(2,3-Dimethylphenyl)-2-(tetrazolo[1,5-a]quinoxaline-4-ylthio)acetamide (3k)

Elemental analysis \% found C 51.82,H 2.99,N 22.66,Cal C 51.75,H 2.87,N 22.58, FT-IR 
$(\mathrm{KBr})$ in $\mathrm{cm}^{-1} 3240(\mathrm{NH}), 3050(\mathrm{C}-\mathrm{H}), 1600(\mathrm{C}=\mathrm{N}), 1030(\mathrm{C}-\mathrm{Cl}), 640\left(\mathrm{CH}_{2}-\mathrm{S}\right){ }^{1} \mathrm{H}$ NMR (DMSOd6, $400 \mathrm{MHZ}$ ppm) 2.745(s,3H,CH$)_{3} 4.442\left(\mathrm{~s}, 2 \mathrm{H}, \mathrm{CH}_{3}\right), 9.166(\mathrm{~s}, 1 \mathrm{H}, \mathrm{N}-\mathrm{H})$.

\section{$N$-(4-Bromophenyl)-2-(tetrazolo[1,5-a]quinoxaline-4-ylthio)acetamide (3l)}

Elemental analysis \% found C 51.82,H 2.99,N 22.66,Cal C 51.70,H 2.85,N 22.54,FT$\operatorname{IR}(\mathrm{KBr})$ in $\mathrm{cm}^{-1}, 3260(\mathrm{~N}-\mathrm{H}), 3040(\mathrm{C}-\mathrm{H}), 2880\left(-\mathrm{CH}_{2}-\right), 1660(\mathrm{C}=\mathrm{O}), 1600(\mathrm{C}=\mathrm{N}), 1420$ and $1250(-\mathrm{C}-\mathrm{S}-(\mathrm{C}-\mathrm{H})), 2880\left(-\mathrm{CH}_{2}-\right), 1660(\mathrm{C}=\mathrm{O}) 1600(\mathrm{C}=\mathrm{N}), 1420$ and $1250 \quad\left(-\mathrm{C}-\mathrm{S}-\mathrm{CH}_{2}\right)$, 1050(C-Cl aryl chloride) and 680 $\left(\mathrm{CH}_{2}-\mathrm{S}\right) .{ }^{1} \mathrm{H}$ NMR (DMSO-d6,400 MHZ ppm) 2.839 $\left(\mathrm{s}, 3 \mathrm{H}, \mathrm{CH}_{3}\right) 4.065\left(\mathrm{~s}, 2 \mathrm{H}, \mathrm{CH}_{2}\right), 7.218-8.024(\mathrm{~m}, 8 \mathrm{H}, \mathrm{Ar}-\mathrm{H})$ and $8.495(\mathrm{~s}, 1 \mathrm{H}, \mathrm{N}-\mathrm{H})$.

\section{Antimicrobial activity}

The purified derivatives were screened for their antimicrobial activity by means of disc diffusion (Kirby Bauer) technique on some bacteria and fungus such as Bacillus subtilis, Escherichia coli, Klebsiella pneumoniae and Staphylococcus aureus and antifungal activity against, Aspergillus niger, Aspergillus flavus, Trichoderma viride \& Candida albicans by standard method by calculating zone of inhibition. Firstly we obtained dilution for their dissolving solvent, thus $10 \mathrm{mg}$ purified sample dissolve into $10 \mathrm{~mL}$ methanol. 5 Dilutions were prepared to the 5 samples. The each dilution contains concentration of samples is $1 \mathrm{mg} / \mathrm{mL}$ for the antimicrobial study. Kirby Bauer filter paper disk method is used for antimicrobial study. In this test, a number of small, sterile filter paper disks of uniform size $(6 \mathrm{~mm})$ that have each been impregnated with a defined concentration of an antimicrobial agent are placed on the surface of an agar plate previously inoculated with a standard amount of the organism to be tested.

Table 2. Antimicrobial screening of the title compounds (3a-31)

\begin{tabular}{|c|c|c|c|c|c|c|c|c|c|}
\hline \multirow[b]{2}{*}{ 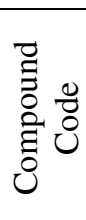 } & \multirow[b]{2}{*}{ 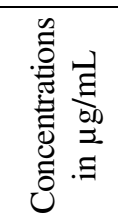 } & \multicolumn{4}{|c|}{ Antibacterial } & \multicolumn{4}{|c|}{ Antifungal } \\
\hline & & 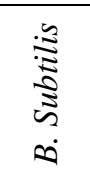 & $\underset{0}{\ddot{0}}$ & 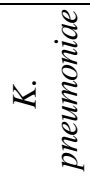 & 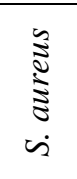 & 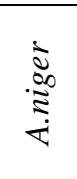 & 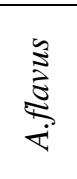 & : & 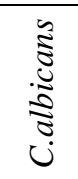 \\
\hline \multirow{5}{*}{$3 \mathbf{i}$} & 1 & 22 & 20 & 19 & 18 & 21.7 & 23.5 & 22 & 21 \\
\hline & 0.5 & 17 & 13 & 11 & 14 & 12.6 & 17.6 & 14 & - \\
\hline & 0.25 & 13 & 12 & 11 & 12 & 11.5 & 13 & 12 & 12.9 \\
\hline & 0.125 & 8 & 7 & 6 & 7.2 & 7.5 & 10.5 & 11 & 6.9 \\
\hline & 0.0625 & 0 & 1 & 2 & 2.1 & 2 & 2 & 2.1 & - \\
\hline \multirow{5}{*}{$3 \mathbf{j}$} & 1 & 20.6 & 19.5 & 17.9 & 16.8 & 22.6 & 15 & 14.9 & 17.4 \\
\hline & 0.5 & 16.8 & 14.8 & - & 17 & 16.4 & 11 & 13 & 11 \\
\hline & 0.25 & 13.7 & - & 12.8 & - & - & 10 & 11 & 12.8 \\
\hline & 0.125 & 7.9 & 9.8 & - & 10.7 & 9.8 & 8.9 & - & 6.8 \\
\hline & 0.0625 & 1.9 & 1.2 & 1.4 & 1.4 & 1.3 & - & 1.1 & - \\
\hline \multirow{5}{*}{$3 \mathbf{k}$} & 1 & 16 & 17 & 16.9 & 12 & 18.9 & 14 & 14.6 & 17.1 \\
\hline & 0.5 & 15 & 13 & 16.7 & - & 15.4 & - & 11.9 & 12 \\
\hline & 0.25 & 13 & 15 & 12.4 & 10.4 & 13.7 & 9.3 & 10.8 & 10 \\
\hline & 0.125 & 7.5 & - & 6.8 & 5.5 & 8.8 & 7.7 & - & 6.6 \\
\hline & 0.0625 & 1.2 & 1 & 1.2 & 1.5 & - & - & 1.1 & - \\
\hline \multirow{5}{*}{31} & 1 & 18.6 & 18.9 & 16.8 & 17.8 & 19.1 & 15 & 21.6 & 16.7 \\
\hline & 0.5 & 16.3 & 12.6 & 15.5 & 16.9 & 15.9 & 13.7 & 14 & 12.8 \\
\hline & 0.25 & 12.5 & 6.3 & - & 8.5 & 13.8 & 5.6 & 8.7 & 11.9 \\
\hline & 0.125 & 7.2 & 9 & 8.6 & 10.5 & 7.6 & 4.5 & - & 6.4 \\
\hline & 0.0625 & 1.2 & - & 1.1 & 1.4 & 1.3 & - & 1.1 & - \\
\hline
\end{tabular}




\section{Conclusion}

In the present investigation $N$-(Substituted phenyl)-2-(tetrazolo[1,5-a]quinoxaline-4-ylthio) acetamide 3(i-l) have been synthesized and characterized by spectral analysis. They all were screened preliminary antimicrobial activity. The electronic factors exerted by the substituted and the hydrophobic nature of phenyl nucleus in the title compounds influenced the activity. In this paper Lipinski's rule of five were calculated. This rule is widely used as a filter for drug like properties. So, none of the compounds violated Lipinski's parameter making them potentially promising agents for antimicrobial therapy.

\section{Acknowledgement}

The authors acknowledge thanks to, Sophisticated Instrument facility Division(SAIF), for providing elemental analysis and NMR spectral data.Authors also admit their thankfulness to Head Department of Chemistry Dr.H.S.Gour University, Sagar for providing I.R. other necessary facilities and also thankful to scan laboratory Bhopal for providing antimicrobial data.

\section{References}

1. Ratnadeep V Ghadage and Pramod J Shirote, Int J Experimental Pharmacology, 2012, 2(1), 44-49.

2. Tandon V K, Yadav D B, Maurya H K, Chaturvedi A K and Shukla P K, Bioorg Med Chem., 2006 , 14(17), 6120-6126; DOI:10.1016/j.bmc.2006.04.029

3. Sarges R, Howard H R, Browne R G, Label L A, B. Kenneth Koe and Seymour P A, J Med Chem., 1990, 33(8), 2240-2254; DOI:10.1021/jm00170a031

4. Sakata G, Makino K, and Kurasawa Y, Heterocycles, 1988, 27(10), 2481-2515; DOI:10.3987/REV-88-397

5. Arthur G, Elor K B, Robert G S, Guo Z Z, Richard J P, Stanley D R, John K, Sean T J, et al., J Med Chem., 2005, 48(3),744-752; DOI:10.1021/jm0492958

6. George D M, Larry D B, John M K, Timothy P B and Braulio S, Bioorg Med Chem Lett., 1997, 7, 2819

7. Szekelyhidi Z, Pato J,Waczek F, Banhegyi, Hegymegi-Barakonyi B, Eros D, Meszaros G, Hollosy F, Hafenbradl D, Obert S, Klebl B, Keri G and Orfi L, Bioorg Med Chem Lett., 2005, 15(13), 3241-3246; DOI:10.1016/j.bmcl.2005.04.064

8. $\quad$ Seitz L E, Suling W J,and Reynolds R C, J Med Chem., 2002, 45(25), 5604-5606; DOI:10.1021/jm020310n

9. Zaragoza F and Stephenson H, J Org Chem., 1999, 64(7), 2555-2557; DOI:10.1021/jo982070i (b) Wu Z and Ede N J, Tetrahedron Lett., 2001, 42(45), 8115-8118; DOI:10.1016/S0040-4039(01)01733-6

10. Sakata G, Makino K and Kurasawa Y, Heterocycles, 1988, 27, 2481-2515; DOI:10.3987/REV-88-397 (References cited therein).

11. Ziegler F E, Comprehensive Organic Synthesis, Combining $C-C \pi$ Bonds, Vol. V, Paquette L A, Ed., Pergamon Press, Oxford, 1991.

12. Ali M M, Ismail M M F, El-Gabby M S A, Zahran M A and Ammar T A, Molecules, 2000 , 5(6), 864-873; DOI:10.3390/50600864

13. Sarges R, Howard R C, Label L A and Seymore P A, J Med Chem., 1990, 33(8), 2240-2254; DOI:10.1021/jm00170a031

14. Mohammad R I and Zahra H, ARKIVOC, 2008, 280-287.

15. Rajurkar R M, Agrawal V A, Thonte S S and Ingale R G, Pharmacophore, 2010, 1(2), 65-76. 
16. Santos T, Salas C R, Colorado P R, Pena H A, Sanchez R A and Flores P A, ARKIVOC (v), 2008, 187-199.

17. Wagle S, Adhikari A V and Kumari N S, Indian J Chem., 2008, 47B, 439.

18. Ajani O, Craig A, Obafemi O and David A Akinpelu, Bioorg Med Chem., 2010, 18(1), 214-221; DOI:10.1016/j.bmc.2009.10.064

19. Singh D P, Deivedi S K, Hashim S R and Singhal R G, Pharmaceuticals, 2010, 3(8), 2416-2425; DOI:10.3390/ph3082416

20. Kumar A, Verma A and Chawla G and Vaishali, Int J Chem Tech Res., 2009, 1(4), $1177-1181$.

21. Khan S, Mullick P, Pandit S and Kaushik D, Pharmaceutical-Drug Research, 2009, 66(2), 169-172.

22. Dell A, William D H, Morris H. R,Smith G. A, Feeney J and Roberts G C K, J Am Chem Soc., 1975, 97(9), 2497-2502; DOI:10.1021/ja00842a029

23. Bailly C, Echepar S, Gago F and Waring M, J Anti-Cancer Drug Des., 1999, 14, 291-303.

24. Richa Shahu and Srivastava S P, Journal of Applicable Chemistry, 2014, 3(4), 1475-1480. 